\title{
Competitive adsorption of multiple proteins to nanoparticles: The Vroman effect revisited
}

\author{
Stefano Angioletti-Uberti ${ }^{\mathrm{a}, \mathrm{b}}$ and Matthias Ballauff ${ }^{\mathrm{c}, \mathrm{d}, \mathrm{e}}$ and Joachim Dzubiella ${ }^{\mathrm{c}, \mathrm{d}, \mathrm{e}, \mathrm{f}}$

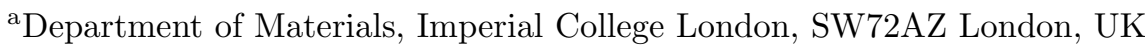 \\ ${ }^{\mathrm{b}}$ Beijing Advanced Innovation Centre for Soft Matter Science and Engineering, Beijing \\ University of Chemical Technology, 100099 Beijing, PR China \\ 'Institut für Physik, Humboldt-Universität zu Berlin, 12489 Berlin, Germany \\ ${ }^{\mathrm{d}}$ Institut für Weiche Materie und Funktionale Materialien, Helmholtz-Zentrum Berlin, 14109 \\ Berlin, Germany \\ ${ }^{\text {e} H e l m h o l t z ~ V i r t u a l ~ I n s t i t u t e, ~ M u l t i f u c t i o n a l ~ B i o m a t e r i a l s ~ f o r ~ M e d i c i n e, ~ K a n t s t r a s s e ~ 55, ~}$ \\ 14513 Teltow, Germany

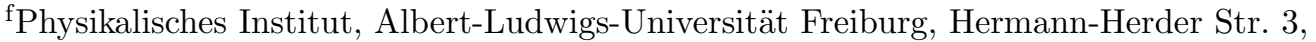 \\ D-79104 Freiburg, Germany
}

\author{
ARTICLE HISTORY \\ Compiled April 6, 2018
}

\begin{abstract}
Proteins adsorbed from the blood plasma change nanoparticles interactions with the surrounding biological environment. In general, the adsorption of multiple proteins has a non-monotonic time dependence, that is, proteins adsorbed at first may slowly be replaced by others. This "Vroman effect" leads to a highly dynamic protein corona on nanoparticles that profoundly influences the immune response of the body. Thus, the temporal evolution of the corona must be taken into account when considering applications of nanocarriers in, e.g., nanomedicine or drug delivery. Up to now, the Vroman effect is explained solely in terms of diffusion: Smaller proteins which diffuse faster are adsorbed first while larger ones, having a stronger interaction with the surface, are preferred at equilibrium. Here we use dynamic density functional theory (DDFT) including steric and electrostatic interactions to provide a full model for the temporal evolution of the protein corona. In particular, we demonstrate that proper consideration of all interactions leads to Vroman-like adsorption signatures in widely different scenarios. Moreover, consideration of energetic terms predicts both competitive as well as co-operative adsorption. In this way, DDFT provides a reacher picture of the evolution of the dynamic protein corona.
\end{abstract}

\section{KEYWORDS}

Protein adsorption, Dynamic Density Functional Theory, Hydrogels, Vroman Effect, Theory and Simulations 


\section{Introduction}

The kinetics of protein adsorption is a complex phenomenon whose understanding is of fundamental importance for the development of various technologies, in particular, nanomedicine applications such as biomedical devices, disease diagnosis, biosensing, drug delivery, biofouling, and bioreactors [1-7]. Once placed into the multi-protein mixture that constitutes the blood plasma, nanoparticles acquire a "protein-corona" that fully coats them. This protein corona provides the true biological identity of the nanoparticle, and controls the immune system response to its presence $[8,9]$. As a matter of fact, various processes, e.g., targeting of cells via nanocarriers, are strongly influenced by the protein corona [10-12]. Hence, it is clear that knowledge of its composition as well as of its evolution with time is an important factor to understand how these nanoparticles interact with the body.

Decades ago, Leo Vroman noted that the adsorption of a mixture of proteins to surfaces displays a peculiar non-monotonicity in time $[4,13]$. Indeed, proteins appearing in the corona at earlier times are often replaced by other, typically larger ones when equilibrium is ultimately achieved. This is a general effect, observed for both flat as well as highly curved surfaces such as those encountered in nanoparticles. To explain this observation, Vroman suggested it was based on the competition between kinetics and thermodynamics: Smaller proteins diffuse faster to the surface but larger ones have a stronger attraction to it and thus dominate at equilibrium. This simple picture, based on ideal diffusion, still dominates the literature[4, 5, 14-16]. However, diffusion of proteins towards a surface is far from ideal and depends on protein-protein mutual interactions as well as the interaction between proteins and the adsorbing surface $[3,17-21]$.

In this paper, we show that when energetic terms are accounted for, Vroman-like, non-monotonic adsorption profiles can be observed under a wide variety of realistic scenarios. These results already strongly question the simple picture provided by Vroman more than half a century ago. Furthermore, we show how a proper characterisation of protein adsorption requires determining the full density profile and not just the amount of proteins adsorbed, as the latter quantity can provide a description masking the true, or better said application-relevant nature of the protein corona. Finally, we also show that protein-protein and protein-nanoparticles interactions not only lead to competitive protein adsorption but in certain scenarios also to cooperative phenomena, where a protein can be more strongly adsorbed to the corona when other proteins are present.

\section{Theoretical model and methods}

Our starting point is the use of dynamic density functional theory (DDFT) [22] to study protein adsorption onto an idealized polymer-coated nanoparticle. We focus on this system because compared to bare nanoparticles, coated ones are much more stable, have freedom in functionalization, and typically have lower toxicities [6, 7, 23-25], and represent the most common type studied in nanomedicine applications.

DDFT can be seen as a generalized diffusion equation with an approximate account of particle-particle correlations [22]. Its use to study protein adsorption has been pioneered by Szleifer and his group [18-20], and is also referred to as the "molecular approach" to diffusion. Compared to the standard diffusion equation, which only considers the Brownian motion of ideal (non-interacting) particles, DDFT also includes 

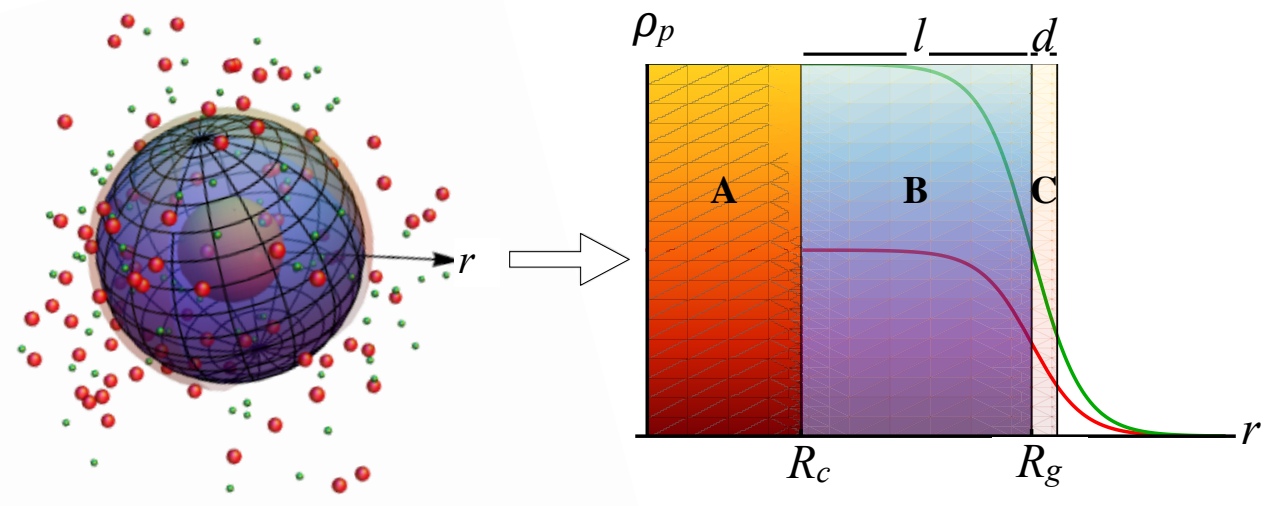

Figure 1. Left: Cartoon representation of our system. A hard-core PMMA nanoparticle (Orange) with radius $R_{\mathrm{C}}=60 \mathrm{~nm}$ is coated with a cross-linked polymer network of PNIPAM $l=90 \mathrm{~nm}$ thick (blue) with an interface width of $d=10 \mathrm{~nm}$ (light orange). This nanoparticle is immersed in a protein solution (green and red spheres). All nanoparticle dimensions are scaled with the correct size ratio, whereas proteins are represented at twice their actual size. Right: The protein density fields for two protein types are represented as continuous, timedependent radial functions $\rho_{p}(r, t)$ (red and green lines). Region $\mathbf{A}$ is the impenetrable hard-core region, $\mathbf{B}$ is the hydrogel region where proteins can adsorb, and $\mathbf{C}$ the hydrogel-bulk interface region (see text).

the interactions within the system in a thermodynamically consistent way. For example, comparisons of DDFT for hard sphere dynamics to particle-based Brownian dynamics computer simulations for radial fluxes have shown excellent agreement for the overdamped dynamics [26]. Our model is depicted in Fig. 1. The nanoparticle is modeled by a core-shell particle of radius $R_{\mathrm{g}}$ with a solid core of radius $R_{\mathrm{c}}$ (region $\mathbf{A}$ in Fig. 1) coated with a thin polymeric surface layer as, e.g., a cross-linked polymer network (hydrogel) with full spherical symmetry. The core is impenetrable to proteins. The surface layer (region $\mathbf{B}$ ) is modeled as a penetrable continuum of width $l$, which is charged due to the presence of ionisable groups. The presence of salt ions in solution enforces overall charge neutrality, leading to a difference in the local electrostatic potential described by the Donnan potential [27]. Since the polymer gel is homogeneous in space, so are all its properties, e.g., the Donnan potential, or the local diffusion coefficient. These properties decay from that of the gel to their value in the bulk solution within an interface width $d$ of a few nanometers [17] (region $\mathbf{C}$ ). This distance is set equal to the average cross-linking distance typically found in the hydrogel $(10 \mathrm{~nm}$ in our simulations). The nanoparticle is immersed in a protein solution, modelled by charged hard spheres of radius $R_{p}$, described via a continuous, time-dependent radial density field $\rho_{p}(r, t)$ with origin at the nanoparticle's center-of-mass and $p$ denotes the protein type.

Within the equilibrium DFT framework, the following free-energy functional well de- 
scribes the free-energy of the protein distributions in our system[17]:

$$
\begin{aligned}
\mathcal{F}=\mathcal{F} & {\left[\left\{\rho_{p}\right\}\right]=\mathcal{F}^{\text {id }}+\mathcal{F}^{\text {ext }}+\mathcal{F}^{\text {exc }} } \\
& =\mathcal{F}^{\text {id }}+\left(\mathcal{F}^{\text {ads }}+\mathcal{F}^{\text {electro }}\right)+\mathcal{F}^{\text {exc }} \\
& =\mathcal{F}^{\text {id }}+\mathcal{F}^{\text {ads }}+\mathcal{F}^{\text {Born }}+\mathcal{F}^{\text {Don }}+\mathcal{F}^{\text {exc }} \\
& =\sum_{p} \int_{V} k_{\mathrm{B}} T \rho_{p}(\mathbf{x})\left[\ln \left(\frac{\rho_{p}(\mathbf{x})}{\rho_{0}}\right)-1\right] d \mathbf{x} \\
& +\int_{V} \rho_{p}(\mathbf{x}) V_{p}^{\text {ads }}(\mathbf{x}) d \mathbf{x}+\int_{V} \rho_{p}(\mathbf{x}) V_{p}^{\text {Born }}(\mathbf{x}) d \mathbf{x} \\
& +\int_{V} z_{p} \rho_{p}(\mathbf{x}) V^{\text {Don }}\left[\left\{\rho_{p}(\mathbf{x})\right\}^{*}\right] d \mathbf{x} \\
& +\int_{V} \rho_{p}(\mathbf{x}) \epsilon^{\text {exc }}\left(\left\{\rho_{p}(\mathbf{x})\right\}\right) d \mathbf{x},
\end{aligned}
$$

where the sum is over all $p$ protein species and the integral has to be read as a threedimensional integral over the whole volume $V$. In Eq. (1), $\rho_{0}$ is a reference density, whose exact value alone is irrelevant. However, the product $\rho_{0} \exp \left(\beta \mu_{p}^{\mathrm{eq}}\right), \mu_{p}^{\mathrm{eq}}$ being the equilibrium chemical potential in the bulk solution, is determined by mass conservation in the system, see [17] for a more detailed discussion. The asterisk in the definition of $V^{\text {Don }}$ means that when calculating its contribution to the chemical potential $\mu_{p}$ by taking the functional derivative, see Eq.(12), this should be done at a fixed value of $V^{\text {Don }}$ to account for the charge-neutrality condition (see the SI for an extended discussion).

Although we refer the reader to our previous publication [17] for details of the implementation and for the solution of the relevant equations, for the sake of clarity we also briefly discuss the origins of this functional here. The first term in Eq.(1), $\mathcal{F}^{\text {id }}$, is the free-energy density for an ideal gas of particles, the second $\mathcal{F}^{\text {ext }}=\mathcal{F}^{\text {ads }}+\mathcal{F}^{\text {electro }}$ describes the coupling between the protein density and an external potential. Following the recipe in [17] this external potential is split into two contributions, one arising from electrostatics, and one from a system-specific adsorption potential $V^{\text {ads }}$ that acts in the gel region $\left(r<R_{g}\right)$. For this term, we choose the simple form:

$$
\begin{aligned}
V_{p}^{\mathrm{ads}}(r) & =S(r) \Delta G_{p}^{\mathrm{ads}} \\
S(r) & =\left[1-\mathrm{Fe}\left(r, R_{g}, d\right)\right] .
\end{aligned}
$$

where $\Delta G_{p}^{\text {ads }}$ is the constant intrinsic adsorption energy per protein and $S$ a switching function, describing the change of environment from that of the bulk gel to that of the bulk protein solution, where $\operatorname{Fe}(r, \mu, \alpha)=1 /(1+\exp [(r-\mu) / \alpha])$ is the Fermi function with inflection point at $\mu$ and width $\alpha$, and $r$ measures the distance from the centre of the nanoparticle. In our model, we take $\alpha$ to be equal to the interface region $d=10 \mathbf{n m}$, as defined in Fig. 1 . The quantity $\Delta G_{p}^{\text {ads }}$ compounds all non-electrostatic interactions between proteins and the polymer coating into a single parameter, and thus includes van-der-Waals attraction, hydrophobic and hydrophilic interactions. In general, this term can be obtained by fitting titration calorimetry data from protein adsorption experiments on mono-component protein solutions [25, 28]. The electrostatic term $\mathcal{F}^{\text {electro }}$ can be further split into two terms: one, $\mathcal{F}^{\text {Born }}$ describ- 
ing the changes in the electrostatic self- (Born) energy and the other, $\mathcal{F}^{\text {Don }}$, coming from the Donnan potential, both dependent on the monopole charge $z_{p}$ of proteins of type $p$, modeled as charged hard spheres $[17,28]$. Their respective form is:

$$
\begin{aligned}
e \beta V^{\text {Don }}(\mathbf{x}) & =\tilde{V}^{\text {Don }}(\mathbf{x})=\ln \left[\sqrt{y(\mathbf{x})^{2}+1}+y(\mathbf{x})\right], \text { with } \\
y(\mathbf{x}) & =\frac{z_{g} \rho_{g}^{c}(\mathbf{x})+\sum_{p} z_{p} \rho_{p}(\mathbf{x})}{2 e \rho_{s}^{\text {bulk }}}
\end{aligned}
$$

where $\rho_{g}^{c}(\mathbf{x})$ and $z_{g}$ are the number density of charged monomers (i.e., $\rho_{g}^{c}=f_{c} \rho_{g}(\mathbf{x})$, where $f_{c}$ is the fraction of charged monomers and $\rho_{g}$ the number density of gel's monomers) and the monomer charge, respectively. Correspondingly, $\rho_{s}^{\text {bulk }}$ is the bulk concentration of salt, $e$ the elementary charge, and finally $z_{p}$ is the charge of a protein of type $p$. As usual, $\beta=1 / k_{\mathrm{B}} T$ where $k_{\mathrm{B}}$ is Boltzmann's constant and $T$ is temperature. The value of $\beta$ is fixed to one, determining our unit for energies. Note that the latter form for the Donnan potential, Eq. (5), is only valid for symmetric monovalent salt. For the Born potential we have instead the form:

$$
\begin{aligned}
\beta V_{p}^{\text {Born }}(\mathbf{x}) & =\frac{z_{p}^{2} \lambda_{\mathrm{B}}}{2 \sigma_{p}} \frac{\kappa(\mathbf{x}) \sigma_{p}}{\left(1+\kappa(\mathbf{x}) \sigma_{p}\right)} \\
\kappa(\mathbf{x}) & =\sqrt{4 \pi \lambda_{B} I_{\text {local }}(\mathbf{x})} \\
& =\sqrt{4 \pi \lambda_{B}\left(z_{g}^{2} \rho_{g}^{c}(\mathbf{x})+z_{s}^{2} \rho_{s}(\mathbf{x})\right)} \\
\rho_{s}(\mathbf{x}) & =\rho_{s}^{\text {bulk }}\left(e^{\left.-\tilde{V}^{\operatorname{Don}(\mathbf{x})}+e^{\tilde{V}^{\operatorname{Don}(\mathbf{x})}}\right)}\right.
\end{aligned}
$$

where $\lambda_{\mathrm{B}}=\frac{e^{2}}{4 \pi \epsilon_{0} \epsilon k_{\mathrm{B}} T}$ is the Bjerrum length (taken to be $0.7 \mathrm{~nm}$ in water at room temperature), $\sigma_{p}$ the radius of protein $p$ and $\kappa(\mathbf{x})$ is the position-dependent inverse screening length which depends on the local ionic strength $I_{\text {local }}(\mathbf{x})=\left[z_{g}^{2} \rho_{g}^{c}(\mathbf{x})+z_{s}^{2} \rho_{s}(\mathbf{x})\right]$ due to the gel and salt ions. In Eq. (7), $z_{g}=-1$ is the valency of the gel, whereas $z_{s}= \pm 1$ that for a monovalent salt. As for the adsorption energy, we assume the polymer density $\rho_{g}$, and hence $\rho_{g}^{c}$, to decay to zero with the functional form of Eq.(3). The salt charge density instead is again dictated by local charge neutrality, consistently with our previous choice of the Donnan potential to describe the electrostatic energy in the system. At this point, it is important to make a comment about the electrostatic part of our functional. A more exact treatment could use a Coulomb functional, at least in a mean-field approximation [26], depending explicitly on the charge density field of all ions in the system, not just proteins. However, this would make studying the dynamics of the system much more complicated and computationally expensive. Instead, we simplify our description using a simple assumption. Considering that monoatomic salt ions are much faster than proteins, we can consider the former to be always in equilibrium w.r.t. the instantaneous position of the latter. Considering a Poisson-Boltzmann description of electrostatics, this also means that on length-scales larger than $1 / \kappa$ the system can be taken to be electrostatically neutral. As we show in a previous publication by some of us (Ref.[28], Appendix A), this assumption leads exactly to the form of the Donnan 
and Born potential that we employ here, including the somewhat peculiar form of a position-dependent inverse screening length depending on both gel and salt ions. Importantly, the electroneutrality assumption (and thus its consequences) are increasingly accurate the lower the protein density in the polymer and the higher the salt concentration (i.e. for shorter screening lengths). Low loadings and relatively high salt-concentration, of the kind found at physiological conditions and hence relevant for biomedical applications, are the regime typically sampled in experiments of protein adsorption on polymer-coated nano- and micro-particles, see e.g. Ref. [29]. Finally, the last term $\mathcal{F}^{\text {exc }}$, typically called the "excess" free-energy, describes proteinprotein interactions. Within our description, these are purely steric in nature and we approximate them within the $B_{2}$ approximation to the hard-sphere interaction within the local density approximation (LDA)[30]. For the packing densities encountered in our system, use of this approximation compared to the full Carnahan-Starling hard sphere functional does not lead to any appreciable difference. Explicitly, this choice results in the following expression:

$$
\begin{aligned}
\mathcal{F}^{\mathrm{exc}} & =\sum_{p} \int_{V} \epsilon^{\mathrm{exc}}\left(\left\{\rho_{p}(\mathbf{x})\right\}\right) \rho_{p}(\mathbf{x}) d \mathbf{x} \\
& =k_{\mathrm{B}} T \sum_{i, j} B_{2}^{i j} \int_{V} \rho_{i}(\mathbf{x}) \rho_{j}(\mathbf{x}) d \mathbf{x},
\end{aligned}
$$

where the indices $i$ and $j$ run over all protein types in the system. To keep the model as simple as possible, we treat protein as spherical, for which a reasonable value for $B_{2}$ is that for hard-spheres of the same mean size [28], giving:

$$
B_{2}^{i j}=\frac{2 \pi}{3}\left(\frac{\sigma_{i}+\sigma_{j}}{2}\right)^{3}
$$

where $\sigma_{i}\left(\sigma_{j}\right)$ is the effective hard-core diameter of protein $i(j)$. In order to account also for polymer-protein excluded volume interactions, one should in principle include in the sum in Eq.(9) one term depending on the polymer density $\rho_{\mathrm{g}}$. We treat instead the polymer as a fixed effective excluded volume zone and scale all protein densities $\rho_{p}$ in Eq.(9) using the following formula:

$$
\rho_{p}(\mathbf{x}) \rightarrow \xi(\mathbf{x}) \rho_{i}(\mathbf{x})=\left(\frac{1}{1-\rho_{g}(\mathbf{x}) v_{\mathrm{mono}}}\right) \rho_{p}(\mathbf{x})
$$

where $v_{\text {mono }}$ is the effective volume of a monomer, approximately $0.3 \mathrm{~nm}^{3}$ in our system [28]. In this way, an increase of the number density of proteins of approximately $8 \%$ is observed in the gel, whereas no scaling occurs outside of it.

Although highly simplified, the use of this particular splitting of the free-energy to describe protein kinetics and thermodynamics has been validated by comparison with experimental data on both single and multi-component protein mixtures [17, 28, 29], with which semi-quantitative agreement was found. Finally, the dynamics of the system is obtained using Eq.(1) together with the following relations: 


$$
\mu_{p}=\frac{\delta \mathcal{F}\left[\left\{\rho_{p}\right\}\right]}{\delta \rho_{p}}
$$

and

$$
\begin{aligned}
\frac{\partial \rho_{p}}{\partial t} & =\nabla \cdot D_{p}\left[\beta \mu_{p}^{\mathrm{id}}+\beta \mu_{p}^{\mathrm{exc}}\right] \\
& =\nabla \cdot D_{p} \nabla \rho_{p}+\nabla \cdot D_{p} \rho_{p} \nabla \beta \mu_{p}^{\mathrm{exc}},
\end{aligned}
$$

Eq.(12) expresses the chemical potential $\mu_{p}$ of species $p$ as a functional derivative of the free-energy functional, as in classical DFT [31]. Eq. (13) instead is simply a generalized diffusion equation for interacting systems [22]. The individual protein diffusion constants are defined by $D_{p}$. We solve Eq. (13) numerically within a cell model, using reflective boundary conditions to enforce number conservation in the system. An extensive and more complete description of all the energy terms and their derivation, as well as the numerical details and techniques used to solve these equations can be found in Ref. [17]. Using this numerical model, we investigate the simplest yet representative protein mixture, comprising two components only, which will be referred to as type 1 and type 2 , that is $p=1,2$.

\section{Results and Discussion}

The values of the parameters entering our dynamical equations for different cases are summarised in Table 1 . These parameters are taken within the range typically measured for different proteins at various values of $\mathrm{pH}$ and salt concentration [28, 29].

\begin{tabular}{|c|c|c|c|c|}
\hline & $z_{p}(e)$ & $\begin{array}{c}\Delta G_{p}^{\text {ads }} \\
\left(k_{\mathrm{B}} T\right)\end{array}$ & $\begin{array}{c}D_{p} \\
\left(\mathrm{~nm}^{2} / \mathrm{ns}\right)\end{array}$ & $\begin{array}{c}\rho_{p}^{\text {bulk }} \\
\left(10^{-4} \mathrm{M}\right)\end{array}$ \\
\hline $\begin{array}{c}\text { a) "Classical" } \\
\text { Vroman }\end{array}$ & $0 / 0$ & $\mathbf{- 1} / \mathbf{- 4}$ & $\mathbf{0 . 0 5} / \mathbf{0 . 0 2}$ & $2 / 2$ \\
\hline $\begin{array}{c}\text { b) "Density" } \\
\text { Vroman }\end{array}$ & $0 / 0$ & $\mathbf{- 2 / - 5}$ & $0.03 / 0.03$ & $\mathbf{3} / \mathbf{1}$ \\
\hline $\begin{array}{c}\text { c) "Charge" } \\
\text { Vroman }\end{array}$ & $\mathbf{2 / 7}$ & $0 / 0$ & $\mathbf{0 . 1} / \mathbf{0 . 0 5}$ & $2 / 2$ \\
\hline $\begin{array}{c}\text { d) "Mixed" } \\
\text { Vroman }\end{array}$ & $\mathbf{3} / \mathbf{2}$ & $\mathbf{0 / - 3}$ & $0.05 / 0.05$ & $2 / 2$ \\
\hline \hline "Co-Adsorption" & $4 /-3$ & $-2 /-5$ & $0.1 / 0.1$ & $2 / 2$ \\
\hline
\end{tabular}

Table 1. Values of the different parameters used in the calculation of Figure 2 (protein type 1 / protein type 2 ). Bold characters are used to highlight the specific parameters differing between the two protein types. 

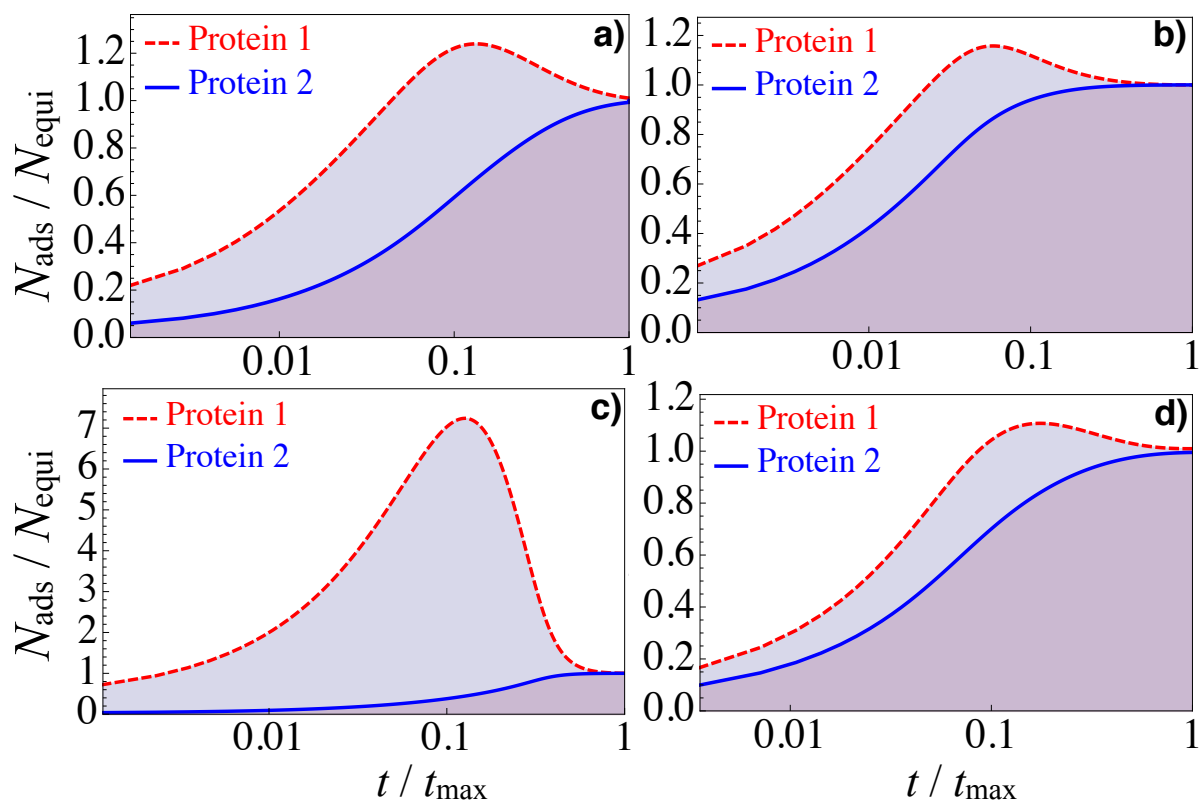

Figure 2. Non-monotonic adsorption profiles arising from different mechanisms (parameters for the simulations in Table 1). For each protein type, we report the amount of protein adsorbed, normalised by their equilibrium value. Time is normalised by the maximum simulation time considered, $t_{\max }$, at which point the system was always found to have reached equilibrium. a) "Classical" Vroman mechanism, where faster protein adsorb first but are then (partially) replaced by more stable ones b) "Density" Vroman, where proteins have the same diffusion coefficient, but different bulk densities and adsorption energy. c) "Charge" Vroman, where one protein has higher diffusion coefficient, but lower charge and d) "Mixed" Vroman, where proteins differ by both their adsorption energy and their charge. In all these cases, competition between different forces lead to a non-monotonic adsorption profile.

In Figure 2, for each protein type we report the number of adsorbed proteins as a function of time $N_{\text {ads }}(t)$, divided by the value attained at equilibrium, $N_{\text {equi }} \equiv$ $N_{\text {ads }}(\infty)$. Formally:

$$
N_{\mathrm{ads}}(t)=4 \pi \int_{R_{\mathrm{c}}}^{R_{\mathrm{g}}+d} \rho_{p}(r, t) r^{2} d r
$$

where $r$ is the distance from the nanoparticle centre, i.e. the adsorbed amount as a function of time, normalised by the adsorbed amount at equilibrium, for four different scenarios. This quantity can be simply calculated by integrating the time-dependent protein density profile inside the gel (i.e. in the interval [0-100] $\mathrm{nm}$ from the hard core of the nanoparticle, see Figs. 1,3 and 4) generated by solving the DDFT equations, Eq.(13). In each case, we normalise the time by the maximum simulation time considered, at which point the system was found to have practically reached equilibrium. Each of the presented cases arises from a representative combination of values for the proteins' charge $z_{p}$, diffusion coefficient $D_{p}$, specific adsorption energy $\Delta G_{p}^{\text {ads }}$ and bulk density $\rho_{p}^{\text {bulk }}$. Note that this latter quantity, although it does not appear explicitly in the equation describing the density field evolution, Eq. 13, is related to the initial density profile at time zero due to number conservation, see [17] for details. An im- 
mediate observation is that all the profiles are qualitatively very similar, despite the fact that the driving forces, as we are about to describe, are quite different for each scenario. We now turn to analyse these cases in more detail. In doing this, we shall also use as a reference Fig. 3 and Fig. 4, where the density profiles and the different terms in the free-energy functional arising from them are plotted for each scenario. For illustrative purposes these profiles are taken at two distinct time points, corresponding roughly to the beginning of the simulation and the time (typically around $\left.t=0.1 t_{\max }\right)$ where protein 1 starts to desorb from the gel. Fig. 2a) describes the "classical" Vroman effect, which our model is indeed able to reproduce. Two proteins with different size, and hence diffusion coefficient, and different specific adsorption energies compete for binding. Indeed, the classical non-monotonic adsorption profile is observed for the smaller (i.e., faster) protein (red line), which is partially replaced at longer times by the more stable one, favoured by a larger adsorption energy $\Delta G_{p}^{\text {ads }}$, as expected at equilibrium. It should be noticed in this regard that this exchange is driven by steric effects enforced by the protein packing penalty included in our DDFT treatment. In absence of any charge, excluded volume interactions are in fact the only term coupling the two protein densities, hence without it each protein would simply adsorb independently.

Fig. $2 \mathrm{~b}$ ) presents a different scenario, the competition between two proteins with the same diffusion coefficient, but differing for their bulk concentration and specific adsorption energy. In this regard, it should be noticed that even for spherical proteins with the same diffusion coefficient, and hence the same size due to Einstein's relation [32], the specific adsorption energy must not be the same, since only the van der Waals contribution should scale with volume but other contributions to $\Delta G_{p}^{\text {ads }}$, e.g. hydrophobic interactions or salt-bridges, do not. Keeping this aspect in mind, let us again describe what we observe in this scenario. The more concentrated protein, type 1 , has a faster initial adsorption but again after reaching a certain maximum value it starts to desorb from the gel, replaced by the one with lower $\Delta G_{p}^{\text {ads }}$. This replacement occurs because of the initial overshooting in the adsorption of protein 1 , which violates the equilibrium condition $\rho_{1} / \rho_{2}=\exp \left[\beta\left(\Delta G_{2}^{\text {ads }}-\Delta G_{1}^{\text {ads }}\right)\right]$, where suffixes indicate the protein type. The faster initial adsorption depends on the higher driving force due to a larger density gradient between the bulk solution and the nanoparticle (only slightly visible in the plot of Fig. 3 because of the scale). Surprisingly, this effect has been overlooked in various discussions on protein adsorption kinetics. In fact, it is not even included in the classical explanation of the Vroman effect, although the concentration of proteins in the blood plasma can differ by orders of magnitude. However, this effect must be expected because different bulk densities generate different density gradients, to which the ideal contribution to the driving force is proportional to. As this gradient relaxes upon adsorption, the (ideal) driving force decreases, and indeed by the time a peak is reached it is even opposing diffusion towards the nanoparticle. Instead, the contribution coming from the specific adsorption energy remains constant and always drives adsorption inside the gel. It is exactly this difference in the timedependence of the driving force that allows the more stable protein to take over if its equilibrium concentration within the nanoparticle polymer coating is favoured by thermodynamics, as we indeed show here.

The previous two examples do not consider any possible electrostatic effect, and the coupling between the diffusion of the two protein is purely due to their excluded volume. For charged systems, however, the overall charge-neutrality condition inside the gel provides an additional parameter connecting them. For proteins of the same charge (and both opposite to that of the gel), competition for adsorption determines a 
Vroman-like profile, see Fig. 2c). In this case, we consider two protein types with the same bulk concentration and no contributions from the specific adsorption energy, although type 1 has a higher diffusion coefficient and a smaller charge compared to type 2. In this case, the replacement of type 1 proteins at larger times can be attributed to a complex competition between global electrostatics, entropy and packing effects. Initially, when proteins adsorb they reduce their concentration gradient with respect to the bulk, i.e., they gain translational entropy. As in the previous two cases, this favours adsorption, with a driving force which is proportional to the protein diffusion coefficient and hence favours a faster adsorption of protein 1 . This ideal contribution decreases with time, and in fact is reversed when the density inside the gel is higher than that in the bulk, see Fig. 4c). At this point, further adsorption requires in our case gaining electrostatic energy. This occurs for both proteins as long as the gel plus protein system is overall neutral (remember the gel is positively charged, whereas the proteins are negatively charged). However, when charge neutrality is achieved it is still possible for the system to lower its free-energy by trading the electrostatic energy of one specie with translational free-energy or, better said, the ideal entropy contribution. In fact, it should be noticed that a protein of higher charge can replace many proteins with smaller ones without changing the overall electrostatic energy, but with an entropy gain due to smoother concentration gradients. In other words, more proteins are free to move in the bulk solution rather than in the restricted region of the gel. Furthermore, this replacement also reduces the overall protein-protein interaction, i.e., the packing (free-)energy, since a smaller number of proteins is required in the core to compensate its charge. Overall, in our system this mechanism leads to a replacement of protein 1 with protein 2 .

In order to show the complexity of protein adsorption even considering a highly simplified model like ours, we finally present in Fig. 2d) an intermediate case between a) and c), whereby the two protein types differ only by their adsorption energy and the charge. The first favours adsorption of type 2 , whereas the second that of type 1 . In this case, the initial faster adsorption of protein 1 is due to the fact that its higher charge compensates for the smaller gradient in adsorption energy. However, and this is crucial, whereas the gradient in the adsorption energy is constant in time, the electrostatic driving force diminishes as the polymer coating is gradually neutralized by the adsorbing proteins. Hence at some point type 1 slows down, and successive replacement is due to a balance between loss of entropy and increase of packing energy (at fixed electrostatic energy) and gain in specific adsorption energy.

Up to this point, we have discussed the Vroman effect analysing the total amount of protein adsorbed in the gel. However, it is arguable that the biological response to the protein corona does not depend on its overall composition, but rather only on that of its "skin", the part effectively exposed to the external environment. Consider for example a cell of the immune system "probing" a nanoparticle by binding to a protein on the nanoparticle's corona. Clearly, in this process only the outer layer is accessible, whereas most of the proteins in the corona will be masked. Whether or not this aspect makes any practical difference depends on spatial density gradients of the protein field in the gel. In this regard, the DDFT formalism is particularly useful, if not crucial. In fact, widely employed theoretical models based on ordinary differential equations only describe the total amount of protein adsorbed [33], but not their spatial profile, and thus would miss any effect due to density inhomogeneities. Instead, having access to the full protein spatial profile allows us to quantify them. This is shown in Fig. 5, where we compare the overall amount adsorbed in the whole gel to that in the outer layer only, defined here as the $d=10 \mathrm{~nm}$ transition region ("skin") between the gel 
and the bulk, see Fig 1 for reference. More precisely, we report the relative fraction $\chi_{p}$ for each specific proteins in the two regions, whose definition is:

$$
\chi_{p}^{\text {skin }}=\frac{\int_{R_{\mathrm{g}}}^{R_{\mathrm{g}}+d} \rho_{p}(r, t) r^{2} d r}{\sum_{p^{\prime}} \int_{R_{\mathrm{g}}}^{R_{\mathrm{g}}+d} \rho_{p^{\prime}}(r, t) r^{2} d r}, \quad \chi_{p}^{\mathrm{gel}}=\frac{\int_{R_{\mathrm{c}}}^{R_{\mathrm{g}}+d} \rho_{p}(r, t) r^{2} d r}{\sum_{p^{\prime}} \int_{R_{\mathrm{c}}}^{R_{\mathrm{g}}+d} \rho_{p^{\prime}}(r, t) r^{2} d r},
$$

Although in both cases one still clearly sees the typical signature of the Vroman effect, the replacement of one protein type with another, the extent of this phenomenon depends on which of the two distinct (albeit spatially overlapping) regions is being considered. For example, such replacement is markedly stronger for the "charge" Vroman case, panel c), then in others. Moreover, the composition of the protein corona in the gel compared to its outmost layer can differ qualitatively and not only quantitatively. In fact the majority component (i.e. that for which $\chi_{p}>0.5$ ) is not even always the same in the two regions. For example, whereas for the "classic" - panel a) - and "charge" - panel c) - scenarios the majority component is the same for both regions, the opposite is true for the "density" -panel b) - and "mixed" - panel d) - cases. Consideration of these differences should be given the proper importance in the analysis of experimental data as well as in their interpretation, especially when considering the biological effects of the protein corona.

The previous examples clearly show that different combinations of the various forces driving protein adsorption can generate competitive effects resulting in very similar non-monotonic profiles as those typically attributed to the "classical" Vroman mechanism. As discussed, understanding these effects requires consideration of the timedependent balance of different energetic terms. This balance, which depends strongly on the protein mixture considered, can lead to other phenomena besides the Vroman effect. In fact, whereas the latter is due to a competition between different proteins, cooperative phenomena, namely co-adsorption, can also occur. This is well illustrated in Fig. 6, where we report the absolute number of protein adsorbed, $N_{\text {ads }}(t)$ in equation (14), for a scenario where proteins have different charges. More precisely, we consider a case where type 2 proteins have a charge of the same sign as that of the polymer and are thus repelled by it. In this case, adsorption in the mono-component mixture is due mainly to a balance between $\Delta G_{p}^{\text {ads }}$, favouring adsorption, and electrostatic energy. The latter still favours adsorption for type 1 but opposes it for type 2 . Hence, in the single-protein solution, a very small amount of type 2 is adsorbed. However, when the two types are mixed, the amount of type 2 adsorbed is two orders of magnitude larger than in the mono-component case. This is not due to any direct attraction between the two protein types because due to their hard core the overall protein-protein interaction in our model is repulsive. What happens instead is that the adsorption of type 1 in the polymer layer reduces the local electrostatic potential, and hence the repulsive force felt by type 2 . The overall result is an almost two orders of magnitude increase in the adsorption of type 2 , hence the term co-adsorption. In this regard, we note that although we use the same term, what we describe is a rather different mechanism from that observed by Vilaseca, Dawson and Franzese in their Molecular Dynamics simulations of protein adsorption on bare surfaces [21]. In their case, co-operative effects arise indirectly because of excluded volume contributions and the fact that two protein types actually compete with a third one. Hence when one of the two displaces their common competitor the second one is also allowed to adsorb. In our case, excluded volume effects at the packing fractions we reach are irrelevant, and co-operation can 
be attributed only to electrostatic coupling. In the future, it would be interesting to test if our more coarse-grained approach can also capture such 3-types packing effects.

\section{Conclusions}

In this paper, we have used DDFT to model adsorption in a bi-component mixture of proteins onto a polymer-coated nanoparticle. We have shown that a correct interpretation of the typical non-monotonic adsorption profiles observed in experiments, the Vroman effect, requires full consideration of the different thermodynamic interactions within the system. In particular, we have shown how completely different mechanisms, physically rationalized by the competition between adsorption energy, electrostatics, and translational and packing entropy, all lead to similar fingerprints. Furthermore, we have shown that a more complete description of this phenomenon requires access to the full spatial density profile of the protein field and not only information of the overall amount adsorbed, as the latter quantity can hide features important to understand the interaction of the protein corona with the biological environment. Finally, we have also shown how inclusion of inter-particle interactions, even at the coarser level of a DDFT description, can lead to the prediction not only of competitive, but also cooperative adsorption, another aspect of clear experimental relevance. In light of these results, design rules to prevent protein adsorption extrapolated from experiments using a single protein type should also be carefully re-assessed [34, 35], since a protein mixture can generate new physics that would not be observed otherwise. Indeed, this might be one of the main reasons why polymer coatings developed to prevent protein adsorption have failed to perform in real-life applications as well as they did in controlled experiments [2]. Finally, we point out that reality can be even more complex than what we described here when multipolar interactions are included, e.g., protein dipoles interacting with local electrostatic fields [36].

\section{5. acknowledgement}

S.A.-U. acknowledges funding from the Beijing Advanced Innovation Centre for Soft Matter Science and Engineering (BAIC-SMSE), PR China. S.A.-U. and J.D. acknowledge also funding from the Alexander von Humboldt $(\mathrm{AvH})$ Foundation via a Post-Doctoral Research Fellowship. Finally, all authors acknowledge support from the Helmholtz Virtual Institute (HVI) "Multifunctional Materials in Medicine" (Berlin and Teltow), Germany, and by the DFG International Research Training Group IRTG 1524 .

\section{References}

[1] K. Nakanishi, T. Sakiyama and K. Imamura, Journal of Bioscience and Bioengineering 91 (3), 233-244 (2001).

[2] Q. Wei, T. Becherer, S. Angioletti-Uberti, J. Dzubiella, C. Wischke, A.T. Neffe, A. Lendlein, M. Ballauff and R. Haag, Angewandte Chemie International Edition 53 (31), 8004-8031 (2014).

[3] S. Angioletti-Uberti, NPJ Computational Materials 3 (1), 48 (2017).

[4] S.L. Hirsh, D.R. McKenzie, N.J. Nosworthy, J.A. Denman, O.U. Sezerman and M.M.M. Bilek, Colloids and Surfaces B: Biointerfaces 103 (0), 395 - 404 (2013). 
[5] S. Choi and J.C. Yongmo Yang, Biosensors and Bioelectronics 24, 893-899 (2008).

[6] K.S. Soppimath, T.M. Aminabhavi, A.R. Kulkarni and W.E. Rudzinski, Journal of Controlled Release 70, 1-20 (2001).

[7] J. Nicolas, S. Mura, D. Brambilla, N. Mackiewicza and P. Couvreura, Chemical Society Reviews 42, 1147-1235 (2013).

[8] M.P. Monopoli, C. Åberg, A. Salvati and K.A. Dawson, Nature Nanotechnology 7 (12), 779-786 (2012).

[9] M.P. Monopoli, D. Walczyk, A. Campbell, G. Elia, I. Lynch, F. Baldelli Bombelli and K.A. Dawson, Journal of the American Chemical Society 133 (8), 2525-2534 (2011).

[10] A. Salvati, A.S. Pitek, M.P. Monopoli, K. Prapainop, F.B. Bombelli, D.R. Hristov, P.M. Kelly, C. Åberg, E. Mahon and K.A. Dawson, Nature Nanotechnology 8 (2), 137-143 (2013).

[11] S. Tenzer, D. Docter, J. Kuharev, A. Musyanovych, V. Fetz, R. Hecht, F. Schlenk, D. Fischer, K. Kiouptsi, C. Reinhardt, K. Landfester, H. Schild, M. Maskos, S. Knauer and R. Stauber, Nature Nanotechnology 8 (10), 772-781 (2013).

[12] A. Lesniak, F. Fenaroli, M.P. Monopoli, C. Åberg, K.A. Dawson and A. Salvati, ACS Nano 6 (7), 5845-5857 (2012).

[13] L. Vroman and A. Adams, Surface Science 16 (0), 438 - 446 (1969).

[14] P. Wojciechowski, P.T. Hove and J.L. Brash, Journal of Colloid and Interface Science 111, 455-465 (1986).

[15] S.Y. Jung, S.M. Lim, F. Albertorio, G. Kim, M.C. Gurau, R.D. Yang, M.A. Holden and P.S. Cremer, Journal of the American Chemical Society 125, 1278212786 (2003).

[16] H. Noh and E.A. Vogler, Biomaterials 28, 405-422 (2007).

[17] S. Angioletti-Uberti, M. Ballauff and J. Dzubiella, Soft Matter 10 (40), 7932-7945 (2014).

[18] I. Szleifer, Biophysical Journal 72 (2 Pt 1), 595 (1997).

[19] J. Satulovsky, M. Carignano and I. Szleifer, Proceedings of the National Academy of Sciences 97 (16), 9037-9041 (2000).

[20] M.A. Carignano and I. Szleifer, Colloids and Surfaces B: Biointerfaces 18 (3 - 4), $169-$ $182(2000)$.

[21] P. Vilaseca, K.A. Dawson and G. Franzese, Soft Matter 9, 6978-6985 (2013).

[22] U.M.B. Marconi and P. Tarazona, The Journal of Chemical Physics 110 (16), 8032-8044 (1999).

[23] C. Röcker, M. Ptzl, F. Zhang, W.J. Parak and G.U. Nienhaus, Nature Nanotechnology 4, 577-580 (2009).

[24] D. Hühn, K. Kantner, C. Geidel, S. Brandholt, I.D. Cock, S.J.H. Soenen, P.R. Gil, J.M. Montenegro, K. Braeckmans, K. Müllen, G.U. Nienhaus, M. Klapper and W.J. Parak, ACS Nano 7, 2253-3263 (2013).

[25] N. Welsch, Y. Lu, J. Dzubiella and M. Ballauff, Polymer 54 (12), 2835 - 2849 (2013).

[26] J. Dzubiella and C.N. Likos, Journal of Physics Condensed Matter 15, L147 (2003).

[27] D.I. Devore and G.S. Manning, Biophysical Chemistry 2, 42 (1978).

[28] C. Yigit, N. Welsch, M. Ballauff and J. Dzubiella, Langmuir 28 (40), 14373-14385 (2012).

[29] M. Oberle, C. Yigit, S. Angioletti-Uberti, J. Dzubiella and M. Ballauff, The Journal of Physical Chemistry B 119 (7), 3250-3258 (2015).

[30] J.P. Hansen and I. MacDonald, Theory of Simple Liquids, 4th ed. (, , 2013).

[31] R. Evans, Advances in Physics 28 (2), 143-200 (1979).

[32] A. Einstein, Annalen der Physik 322 (8), 549-560 (1905).

[33] D. Dell'Orco, M. Lundqvist, C. Oslakovic, T. Cedervall and S. Linse, PLoS ONE 5 (6), e10949 (2010).

[34] R.G. Chapman, E. Ostuni, S. Takayama, R.E. Holmlin, L. Yan and G.M. Whitesides, Journal of the American Chemical Society 122 (34), 8303-8304 (2000).

[35] E. Ostuni, R.G. Chapman, R.E. Holmlin, S. Takayama and G.M. Whitesides, Langmuir 17 (18), 5605-5620 (2001).

[36] I. Adroher-Benítez, A. Moncho-Jordá and J. Dzubiella, Langmuir 33 (18), 4567-4577 (2017). 


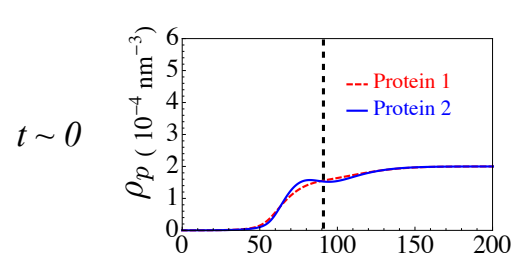

$$
X=\text { - External — Donnan — Excess — Ideal — Born }
$$
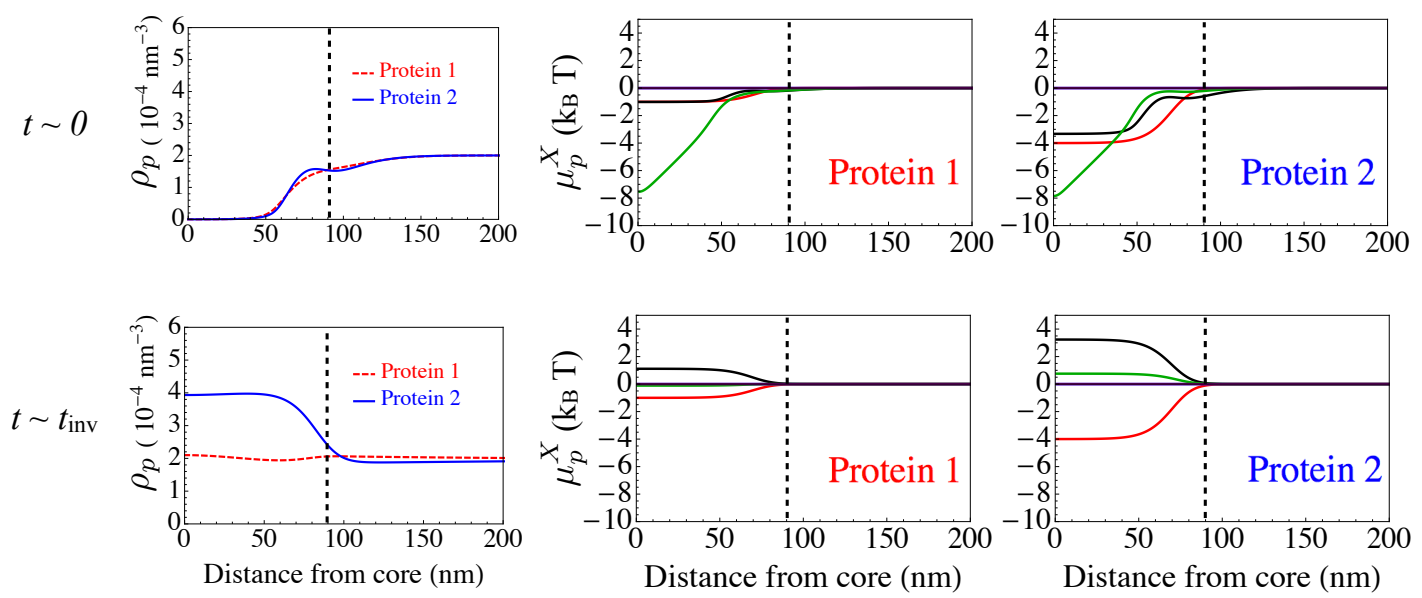

\section{a) Classic Vroman}
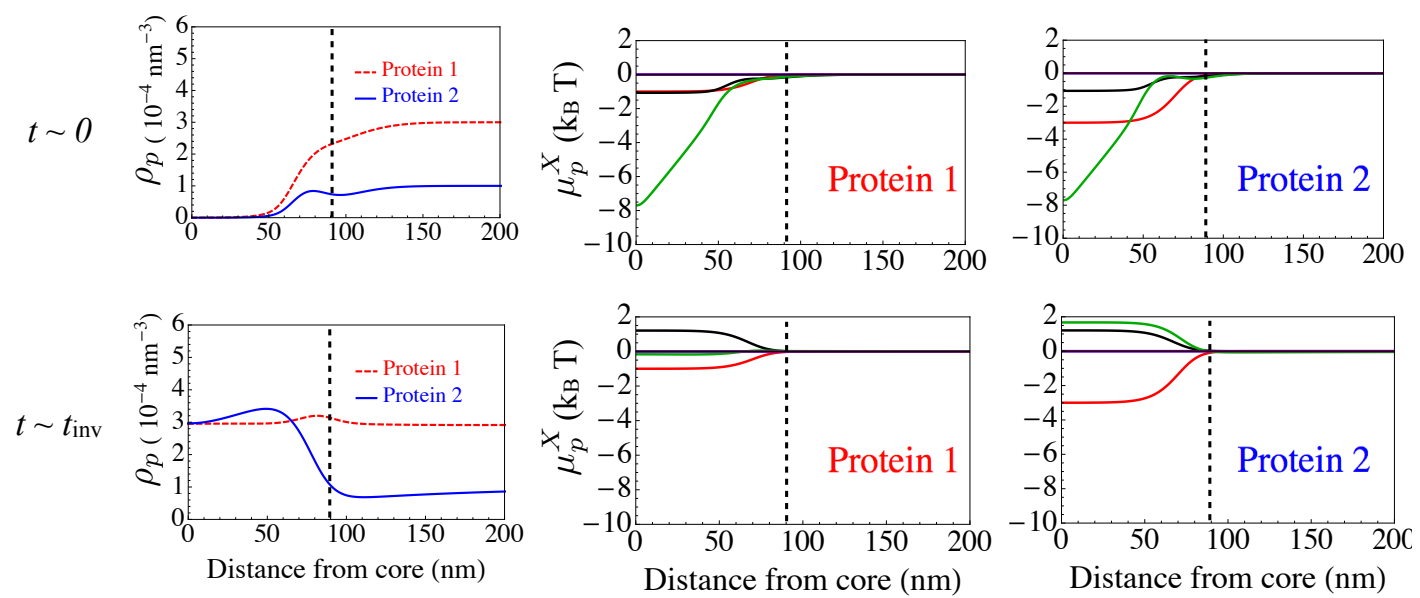

\section{b) Density Vroman}

Figure 3. Density field $\rho_{p}$ (left panels) and contributions to the potential $\mu_{p}^{x}$ (centre and right panels) for the two protein types $\mathrm{P}_{1}$ and $\mathrm{P}_{2}$, respectively, under the different scenarios simulated, see Table 1 . Data are shown at two different time-points, corresponding roughly to the initial time (top panels) and to a time $t_{\text {inv }} \equiv 0.1 t_{\text {max }}$ (bottom panels), where the Vroman effect typically occurs, $t_{\max }$ being the maximum simulated time, at which equilibrium is observed. For reference, the dashed vertical black line is at $r=R_{\mathrm{g}}$, at the boundary of the polymer gel. 

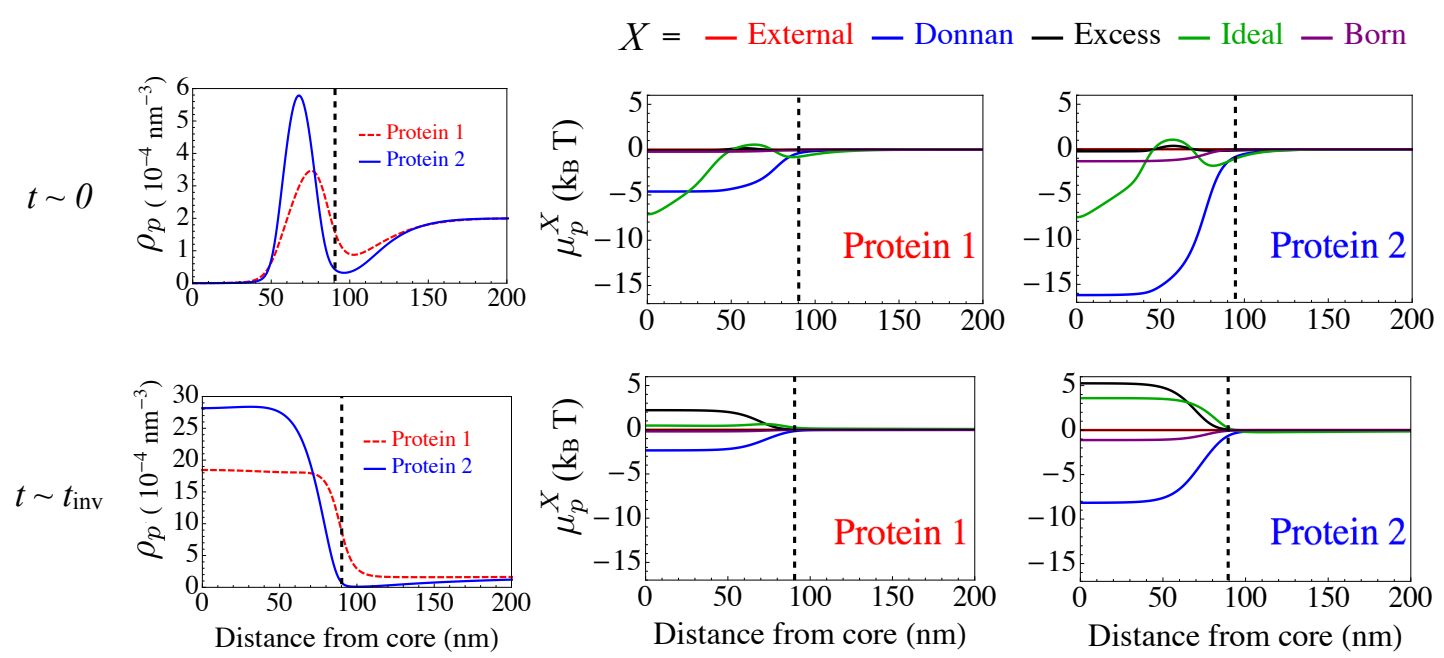

\section{c) Charge Vroman}
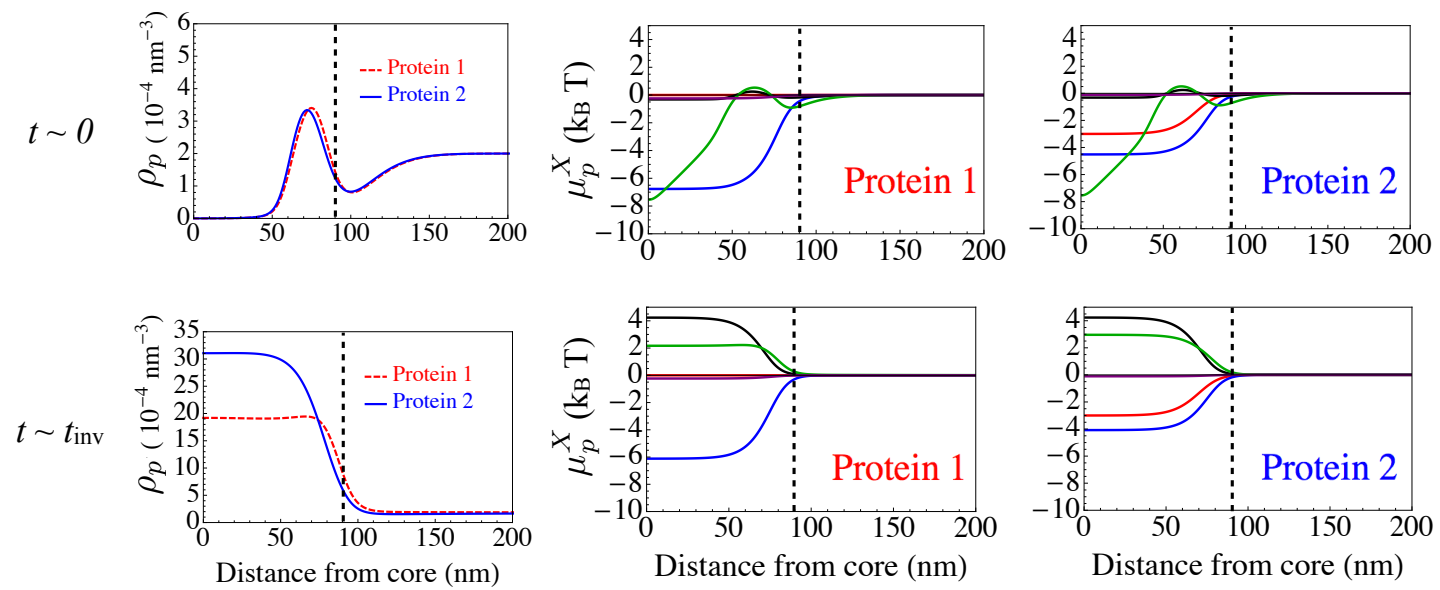

d) Mixed Vroman

Figure 4. As in Figure 3, but for the "Charge Vroman" and "Mixed Vroman" scenario, see Table 1 for reference 
a)

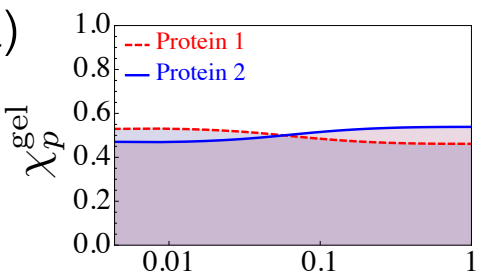

b)

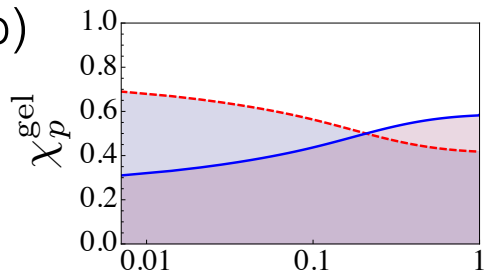

C)

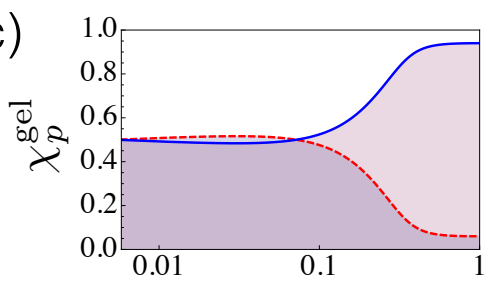

d)

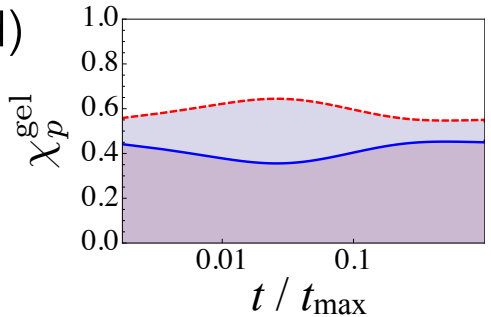

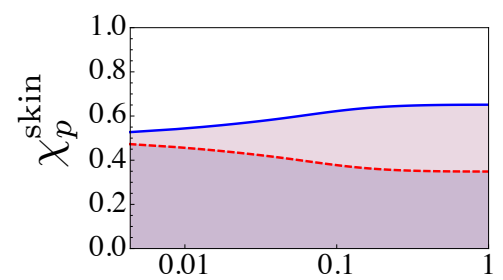
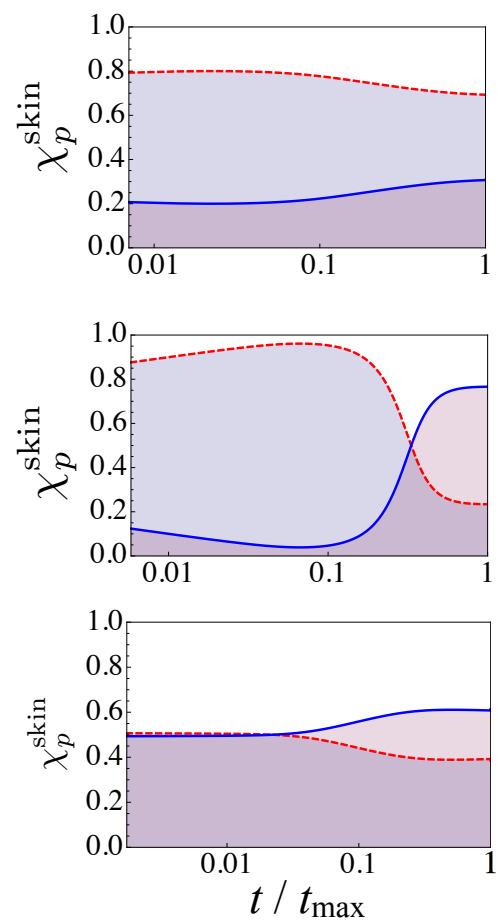

Figure 5. Comparison of the overall composition of the adsorbed proteins $\chi_{p}^{\text {gel }}$ vs the composition of the outmost layer $\chi_{p}^{\text {skin }}$ of the protein corona, see Eq.(15). Panels are labelled according to the description reported in Table 1. Although the typical signature of the Vroman effect, a replacement of one protein type with another, can be clearly seen in both cases, this replacement is not the same considering the two different (albeit overlapping) regions. 


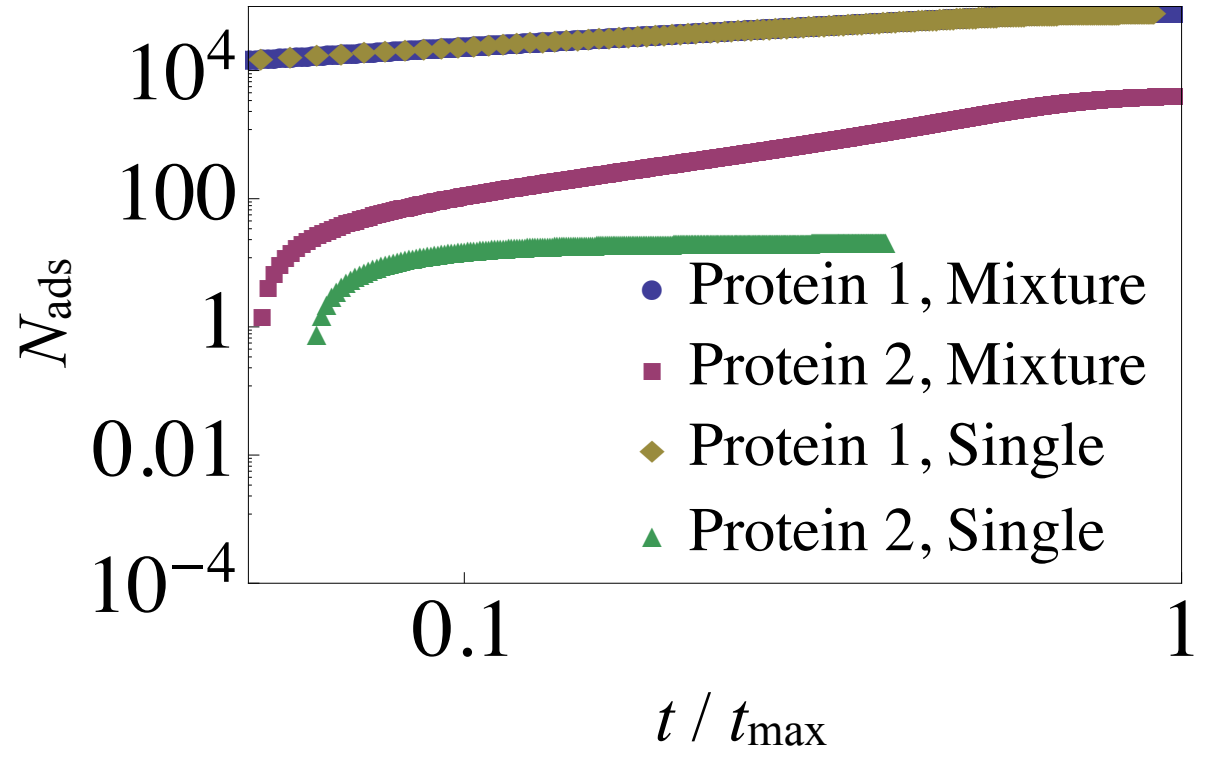

Figure 6. Total number of adsorbed proteins $N_{\text {ads }}$, see Eq.(14) in a bi-component solution (circles and square for protein 1 and 2, respectively ) compared to the case when a single type, with the same amount, is present in solution (diamonds and triangles). Whereas the adsorption profile is very similar to that of protein 1 , adsorption of protein 2 increases by almost two orders of magnitude in the bi-component mixture, due to synergistic effects brought by global electrostatic interactions. 